Artigo de Revisão

\title{
Controle biológico de helmintos parasitos de animais: estágio atual e perspectivas futuras ${ }^{1}$
}

\author{
Marcelo de Andrade Mota ${ }^{2 *}$, Artur Kanadani Campos $^{2}$ e Jackson Victor de Araújo ${ }^{3}$ \\ ABSTRACT.- Mota, M.A., Campos, A.K. \& Araújo, J.V. [Biological control of helminth parasites \\ of animals: Current stage and future outlook. A review.] Controle biológico de helmintos \\ parasitos de animais: estágio atual e perspectivas futuras. Pesquisa Veterinária Brasileira 23(3):93- \\ 100. Depto Parasitologia, Universidade Federal de Minas Gerais, Cx. Postal 486, Belo Horizonte, \\ MG 31270-901, Brazil. E-mail: mmota@icb.ufmg.br \\ Biological control is a non-chemical alternative method with its main goal to reduce the \\ amount of parasite population using natural antagonists. The administration of \\ nematophagous fungi to animals has been considered an alternative in gastrointestinal \\ helminthiasis prophylaxis. The nematophagous fungi produce trap-shaped structures, which \\ are responsible for capturing and destroying the free-living stages of nematodes. The genera \\ Arthrobotrys, Duddingtonia and Monacrosporium has been shown efficacy in laboratory and \\ field experiments against cattle, equine, ovine and swine parasites. Several fungi formulations \\ have been evaluated, but there is so far no commercial product available. The association \\ of research groups with industry could improve the successful implementation of this control \\ method.
}

INDEX TERMS: Biological control, gastrointestinal helminths, prophylaxis.

RESUMO.- O controle biológico é um método desenvolvido para diminuir uma população de parasitas pela utilização de antagonista natural. A administração de fungos nematófagos aos animais domésticos é considerada uma promissora alternativa na profilaxia das helmintíases gastrintestinais parasitárias. Os fungos nematófagos desenvolvem estruturas em forma de armadilhas, responsáveis pela captura e destruição dos estágios infectantes dos nematóides. Os fungos dos gêneros Arthrobotrys, Duddingtonia e Monacrosporium têm demonstrado eficácia em experimentos laboratoriais e no campo no controle de parasitos de bovinos, equiinos, ovinos e suínos. Diversas formulações fúngicas têm sido avaliadas, no entanto, ainda não há nenhum produto comercial disponível. A associação dos grupos de pesquisa e o envolvimento

${ }^{1}$ Recebido em 24 de abril de 2002.

Aceito para publicação em 21 de março de 2003.

${ }^{2}$ Doutorado em Parasitologia, Universidade Federal de Minas Gerais. Bolsista CAPES. *Endereço para corespondência: Depto Parasitologia, UFMG, Cx. Postal 486, Belo Horizonte, MG 31270-901. E-mail: mmota@icb.ufmg.br

${ }^{3}$ Depto Medicina Veterinária, Universidade Federal de Viçosa, Av. P.H. Rolfs s/n, Campus Universitário, Viçosa, MG 36570-000. das indústrias poderão colaborar para o sucesso na implementação desta forma de controle.

TERMOS DE INDEXAÇÃO: Controle biológico, helmintos gastrintestinais, profilaxia.

\section{INTRODUÇÃO}

Dentre os fatores que interferem no desenvolvimento pleno da atividade pecuária, as helmintíases gastrintestinais ocupam lugar de destaque (Macrae 1993). Os prejuízos estão relacionados ao retardo na produção, custos com tratamentos profilático e curativo e em casos extremos, a morte dos animais. Enquanto nos países desenvolvidos os gastos devidos aos custos com controle são significativos, nos países em desenvolvimento as doenças parasitárias causam prejuízos pela diminuição na produção e na restrição à criação de animais com reduzida susceptibilidade às parasitoses, porém com baixas performances produtivas. As raças de animais com melhores índices produtivos, quase sempre criadas nos países desenvolvidos, raramente se sobressaem em condições ambientais onde há grande disponibilidade de parasitos durante todo ano (Perry \& Randolph 1999).

O conhecimento sobre a epidemiologia dos parasitos e suas interações com os hospedeiros em um determinado ambiente e sistema produtivo são os requerimentos mais 
importantes no estabelecimento de um sistema de controle efetivo. A falta destas informações pode levar a utilização inadequada de tratamentos anti-helmínticos, relacionadas ao rápido desenvolvimento de resistência e traduzida em aumentos de casos clínicos e perdas produtivas.

Os programas de controle parasitários eficientes estão baseados em informações sobre a disponibilidade de larvas no ambiente, detecção de fontes de infecção, conhecimento sobre as exigências climáticas para eclosão de ovos e viabilidade larvar. Medidas preventivas baseadas nestas informações podem diminuir a frequiência de tratamentos químicos e quando associadas a outras formas de controle podem reduzir a dependência dos anti-helmínticos (Stromberg 1997, Barger 1999, Stromberg \& Averbeck 1999).

Esta revisão tem como objetivo apresentar a importância do controle biológico como alternativa promissora para o controle de parasitos de animais domésticos.

\section{CONTROLE ALTERNATIVO DAS HELMINTÍASES GASTRINTESTINAIS}

Os problemas relacionados à resistência e ecotoxicidade enfatizam a necessidade de serem implementados programas integrados de controle parasitário, que assegurem saúde e segurança dos organismos vivos, por meio de tratamentos estratégicos baseados na epidemiologia, eliminação de vermifugações desnecessárias, utilização de pastoreio alternado e higienização de pastagens. Além disto, deve-se evitar o uso continuado de uma mesma classe de anti-helmíntico, assim como a rápida rotação de compostos, a introdução de vermes resistentes e a utilização de doses inferiores às recomendadas.

A identificação de marcadores relacionados à resistência genética dos hospedeiros ao estabelecimento dos parasitos, pode ser um meio de seleção para tomada de decisões sobre cruzamentos raciais. Práticas de manejo que contribuam para o aumento da imunidade, pela nutrição e ou vacinas, também podem ser úteis para incrementar os níveis produtivos. A utilização de taninos extraídos a partir de plantas, que funcionem como vermífugos e a seleção de espécies de gramíneas que dificultem o desenvolvimento larvar no meio ambiente são propostas disponíveis, porém ainda não exploradas e validadas (Rew 1999, Thamsborg et al. 1999, Williams 1999).

\section{CONTROLE BIOLÓGICO DE NEMATÓIDES}

Como regra de manutenção dos sistemas biológicos, toda população é regulada por antagonistas. Este processo ocorre espontaneamente na natureza e não é dependente da interferência do homem. Na ausência de controladores naturais, a população de um determinado organismo poderia aumentar indiscriminadamente.

Normalmente, o termo controle biológico se aplica à utilização de antagonistas naturais disponíveis no ambiente, para diminuir a um limiar sub-clínico e economicamente aceitável a população de um agente causador de perdas produtivas à atividade pecuária ou agrícola (Grønvold et al. 1996). Na prática, o controle biológico não atua sobre estágios internos de parasitos; contudo, concentra suas ações sobre os hospedeiros intermediários, paratênicos, vetores e estágios larvais de vida livre, diminuindo a fonte de infecção para os hospedeiros finais, além disso, causam menos efeitos negativos no ambiente que os métodos químicos.

Os microorganismos selecionados como antagonistas naturais devem possuir especificidade de ação, alta capacidade reprodutiva e suportar as condições ambientais no local em que o controle é realizado. A seleção de um agente que possa ser empregado comercialmente como controlador biológico de parasitos gastrintestinais de ruminantes está baseada na capacidade de produção do antagonista em escala industrial, nos custos relacionados a esta produção, na competitividade com as drogas tradicionais estabelecidas no mercado e no tempo de sobrevivência do organismo em formulações comerciais. Deve-se atentar para que as formulações ofereçam segurança para produtores, consumidores, animais tratados e ao meio ambiente e finalmente, que seja efetivo no controle do organismo alvo (Grønvold et al. 1996).

\section{Fungos nematófagos}

Os fungos nematófagos, normalmente chamados de fungos destruidores de nematóides, estão catalogadas com mais de 150 espécies (Barron 1977). Eles são divididos em três grupos. A maioria das espécies está classificada como fungos predadores de nematóides. Estes fungos produzem estruturas em forma de anéis constritores e não constritores, hifas, botões e redes tridimensionais adesivas ao longo do micélio. $\mathrm{O}$ aprisionamento à armadilha é seguido pela penetração das hifas na cutícula do nematóide. Dentro do nematóide, ocorre o crescimento das hifas e a digestão dos conteúdos internos.

Um segundo grupo, denominados fungos endoparasitos, é capaz de infectar os nematóides através de esporos, que uma vez ingeridos desenvolvem hifas responsáveis pela absorção do conteúdo interno do nematóide. Estes fungos não produzem hifas vegetativas fora do corpo do hospedeiro, mas hifas férteis ou conidióforos contendo esporos.

O terceiro grupo de fungos é denominado oportunistas, parasitos de ovos. As hifas penetram a casca do ovo, através dos pequenos poros existentes na camada vitelínica, causando alteração na permeabilidade da casca e expandindo seu volume. A hifa aumenta de tamanho ao passar pela camada vitelínica e atravessa a camada adjacente quitínica e lipídica. Como consequiência do processo, a camada vitelínica se divide, a camada de quitina se torna vacuolizada e a camada de lipídios se torna dispersa. Hifas endógenas emergem do ovo e produzem conidióforos, funcionando como fonte de conídios. Estes tipos de fungos colonizam o conteúdo do ovo, ou ainda a larva em desenvolvimento no seu interior (MorganJones \& Rodríguez-Kábana 1988).

Fungos predadores de nematóides

Os fungos predadores nematófagos são os organismos antagonistas de nematóides mais pesquisados, pois têm mostrado serem capazes de reduzir efetivamente populações de nematóides em condições de laboratório e no campo (Larsen 1999). A formação de armadilhas, ao longo de suas 
hifas, ocorre em resposta à presença do nematóide ou suas excretas, de compostos biológicos ou ainda, é induzida por condições de estresse fisiológico, como na escassez de nutrientes e água (Balan \& Gerber 1972). O processo de diferenciação das armadilhas ocorre dentro de 24 horas após a interação fungo e nematóide (Pramer 1964). Quanto maior a motilidade dos nematóides, maior o estímulo ao fungo para a produção de armadilhas (Nansen et al. 1986, 1988). A formação de armadilhas pode ser atribuída também aos esporos (Dackman \& Nordbring-Hertz 1992).

Embora muitos fungos predadores de nematóides tenham sido isolados e identificados durante o fim do século dezenove (Zopf 1888), muitas informações sobre as características ecológicas, nutricionais e fisiológicas destes organismos só recentemente foram geradas. No solo, onde prevalecem condições nutricionais estressantes para o desenvolvimento dos fungos, a habilidade em predar nematóides propicia a estes tipos de microorganismos vantagens adicionais de sobrevivência. Algumas espécies desenvolvem estruturas de captura como resultado de estímulos externos, enquanto outras desenvolvem-nas espontaneamente, sendo as mais dependentes de nematóides como fonte de nutrientes.

As espécies formadoras espontâneas de armadilhas são mais abundantes em solo contendo disponibilidade de matéria orgânica e esta habilidade confere a estas espécies vantagem sobre as espécies não espontâneas em solo com fauna e flora microbiana rica (Gray 1985). Alguns experimentos falharam na tentativa de indicar os requerimentos nutricionais para o desenvolvimento de culturas de fungos nematófagos (Coscarelli \& Pramer 1962), porém recentemente foi demonstrado que fungos nematófagos crescem bem em meio de cultivo contendo ácido oléico e o aminoácido D-alanina como fonte de carbono e energia para o processo de formação de armadilhas (Rosenweig 1983, Dijksterhuis et al. 1993). O crescimento rápido e a produção abundante de micélio são dois importantes fatores para a disseminação e sobrevivência dos fungos em condições ambientais, embora o crescimento micelial não esteja relacionado com a capacidade de um isolado em predar nematóides (Dackman et al. 1987).

\section{Interação entre fungos e nematóides}

O papel dos nematóides na nutrição dos fungos nematófagos ainda não é claro. Fungos predadores são capazes de invadir e consumir a carcaça de nematóides em decomposição, porém o processo de invasão é mediado apenas por hifas vegetativas através de orifícios naturais, numa velocidade muito menor do que a invasão mediada por armadilhas em nematóides vivos (Nordbring-Hertz \& Stälhammar-Carlemalm 1978).

Como resultado de estudos de ultra-estrutura do processo de penetração das hifas, descobriu-se um pouco sobre os mecanismos envolvidos na interação dos fungos com os nematóides. $\mathrm{O}$ nematóide é inicialmente mantido aderido ao fungo por meio de uma substância fibrilar adesiva rica em fosfatase ácida, que também é responsável pela degradação da cutícula. $O$ processo de penetração acontece em uma hora e está associado a presença de corpos densos ricos em enzima que atuam como peroxissomos e encontrados apenas nas armadilhas e nunca em hifas vegetativas. Estes corpos são detectados na primeira hora do processo de penetração e depois desaparecem (Jansson \& Nordbring-Hertz 1988).

$O$ fase final de penetração da cutícula parece ser resultado de força mecânica (Veenhuis et al. 1985). A segunda cutícula das larvas infectantes $\left(\mathrm{L}_{3}\right)$ dos nematóides parasitos de animais domésticos, parece não proteger as larvas da predação pelos fungos, pois os mesmos são menos hábeis em predar $\mathrm{L}_{3}$ sem cutícula externa (Wharton \& Murray 1990). Em experimentos mais recentes, entretanto, se verificou que isolados de Arthrobotrys spp tiveram maior capacidade predatória sobre larvas infectantes sem cutícula externa, demonstrando que há uma complexa relação entre receptores de superfície dos nematóides e ligantes presentes na parede celular dos fungos (Araújo 1998).

Fungos utilizados no controle biológico de nematóides parasitos de animais domésticos

Os fungos nematófagos podem apresentar esporos bastante diversificados no tamanho, coloração, forma e resistência no ambiente. A maioria dos fungos nematófagos apresenta esporos secos, emergindo de estruturas de frutificação, denominadas conidióforos, essenciais na dispersão aérea dos conídios. Os conidióforos crescem verticalmente, em direção perpendicular ao substrato o qual o isolado foi cultivado. Algumas espécies produzem conidióforos contendo apenas um conídio em sua extremidade, outras espécies apresentam cachos de conídios em toda a estrutura do conidió-foro. Estruturas denominadas clamidósporos também podem ser produzidas. Estes são esporos de parede espessa, diferenciada a partir das hifas, aparecem em condições de estresse extremo e podem dar origem a hifas, conidióforos e conídios (Barron 1977).

A grande maioria dos fungos nematófagos é mitospóricos e antigamente eram classificados na divisão Deuteromycetes, classe Hyphomycetes, ordem Hyphomycetales e família Moliniaceae. Apresentam micélio septado e bem desenvolvido, reproduzindo-se agamicamente por espóros exógenos, que são formados sobre ramificações do micélio (Drechsler 1937). Até 1964, a maioria dos fungos era classificada como pertencentes aos gêneros Arthrobotrys, Dactylaria, Dactyella e Trichothecium. Posteriormente, vários novos gêneros foram descritos, incluindo Duddingtonia, Monacrosporium, Genicularia e Dactylariopsis (Gray 1987). Recentemente, estágios de reprodução sexuada destes fungos foram observados para algumas espécies que estão sendo reconhecidas como pertencentes ao filo Ascomycota (Griffin 1994).

O gênero Arthrobotrys reúne um grande número de espécies de fungos nematófagos. $\mathrm{O}$ gênero foi descrito por Corda em 1839, originário de amostras de solo, porém nesta época, a capacidade de predar nematóides não foi mencionada. Todas os isolados do gênero são capazes de produzir armadilhas na presença de nematóides de vida livre, como Panagrellus redivivus ou Turbatrix aceti. $\mathrm{O}$ gênero é classificado como nematófago facultativo (Cooke \& Godfrey 1964). As espécies pertencentes ao gênero formam conídios blásticos de até três 
septos, com formato ovóide, proliferando-se na extremidade dos conidióforos. Há abundância na formação de conidióforos e no processo de conidiogênese, iniciado de forma simpodial (Zhang et al. 1996a). A espécie Arthrobotrys robusta possui conidióforo ereto, algumas vezes ramificado, com cerca de $300 \mathrm{~mm}$ de comprimento, com a extremidade do conidióforo aumentada de tamanho, carregando normalmente seis conídios de formato ovóide, hialino, septados próximo a região mediana, medindo $18-27 \mathrm{~mm}$ de comprimento por 8-12 mm de largura. É capaz de produzir clamidósporos e produzem redes adesivas para predar nematóides (Van Oorschot 1985).

As espécies do gênero Monacrosporium são caracterizadas por produzirem apenas um conídio na extremidade do conidióforo. Os conídios são hialinos, fusiformes, com dois a quatro septos transversais, sendo que a célula intermediária é maior que as da extremidade. A espécie Monacrosporium thaumasium preda nematóides por meio de redes adesivas, produz conídios medindo entre $27-49 \mathrm{~mm}$ de comprimento por $15-23 \mathrm{~mm}$ de largura. 0 conidióforo é ramificado próximo a extremidade, onde cada extremidade carrega um conídio (Liu \& Zhang 1994, Zhang et al. 1996b). Normalmente é evidenciada a presença de clamidósporos em culturas de placa com meio de cultura sólido limitante (Saxena \& Mittal 1995).

Para o gênero Duddingtonia as espécies são caracterizadas por produzir vários conídios na extremidade dos conidióforos (Van Oorschot 1985). Os conídios apresentam formato variando entre elíptico e ovóide apresentando um septo mediano. Duddingtonia flagrans é a espécie mais estudada no controle das helmintoses gastrintestinais de animais domésticos. Predam nematóides por meio de hifas adesivas. Produzem conídios com morfologia de $25-50 \mu \mathrm{m}$ de comprimento por 10-15 $\mu \mathrm{m}$ de largura (Cooke \& Godfrei 1964). Produzem grande quantidade de clamidósporos em matéria seca. A maioria dos isolados mantida em laboratório foram isolados de fezes de animais e na matéria orgânica presente no solo.

\section{TESTES DE INTERAÇÃO EM CONDIÇÕES LABORATORIAIS}

Os estudos iniciais utilizando fungos nematófagos como controladores biológicos de parasitos gastrintestinais de animais foram realizados no início do século XX, na França. Estes estudos mostraram a ação de fungos predadores sobre larvas infectantes de Strongyloides papillosus e Bunostomum phlebotomum em condições laboratoriais e no campo (Descazeaux 1939, Descazeaux \& Capelle 1939, Roubaud \& Descazeaux 1941). Recentemente, foram retomados ensaios com diferentes espécies de fungos no combate a diversas espécies de parasitos de animais domésticos, onde esta interação foi observada em placas de Petri preenchidas com meio de cultura. Um dos experimentos pioneiros, observou o efeito antagônico de diversos fungos predadores dos gêneros Arthrobotrys, Dactylaria, Monacrosporium e Trichothecium sobre larvas infectantes de Ostertagia ostertagi e Trichostrongylus axei (Pandey 1973). A avaliação da interação in vitro entre $A$. oligospora e larvas de Cooperia spp e 0 . ostertagi resul- tou na diminuição da população de nematóides e gerou algumas conclusões sobre necessidades de crescimento dos fungos (Grønvold et al. 1985, Grønvold 1989). A capacidade da espécie $D$. flagrans de produzir armadilhas e clamidósporos, assim como a sua taxa de crescimento, em presença de $\mathrm{L}_{3}$ de 0 . ostertagi foi observada, indicando esta espécie como promissor agente no controle biológico (Grønvold et al. 1996). A adição de conídios de $A$. oligospora às fezes de bovinos, infectadas com $S$. papillosus foi capaz de reduzir o número de larvas infectantes (Chandrawathani 1998).

No Brasil, estudos pioneiros, demonstraram que Arthrobotrys spp e Monacrosporium ellipsosporum foram eficazes no controle de larvas de Haemonchus placei (Araújo et al. 1992, 1993). Vários isolados do gênero Arthrobotrys foram testados sobre larvas infectantes de Haemonchus contortus de ovinos, onde se obteve redução significativa de larvas nas placas tratadas em comparação com as placas do grupo controle sem adição de fungo (Mendonza de Gives et al. 1992, 1994). Em um experimento de interação entre larvas infectantes de $H$. contortus, de caprinos e os fungos $M$. thaumasium e Arthrobotrys conoides, observou-se que os dois isolados são capazes de reduzir a população de larvas infectantes, porém a espécie M. thaumasium mostrou ser mais eficiente (Mota et al. 2000). A administração de conídios de $A$. oligospora às fezes de equiino mostrou ser capaz de reduzir a população de larvas infectantes de ciatostomíneos (Charles et al. 1995). As conclusões destes estudos em condições de laboratório foram favoráveis à utilização de fungos como controladores biológicos de larvas de parasitos de animais domésticos (Larsen 1999).

\section{TESTES DE PASSAGEM DOS ISOLADOS PELO TRATO GASTRINTESTINAIS DE ANIMAIS}

Embora haja possibilidades da utilização de fungos nematófagos para o controle das verminoses dos animais domésticos, o melhor local de atividade tem se mostrado ser as fezes frescas, onde todos os nematóides parasitos de rebanho têm que passar desde estágio de ovo até os estágios larvais, antes de migrar para a pastagem como larva infectante. Há necessidade de determinar que espécies fúngicas são capazes de sobreviver a passagem pelo trato gastrintestinal dos animais, germinar nas fezes e capturar larvas infectantes.

A passagem de fungos pelo trato gastrintestinal de animais domésticos e a avaliação da eficácia dos isolados após a passagem tem sido o foco de diversos estudos. A administração de Arthrobotrys superba em grãos de cevada a bovinos infectados com nematóides parasitos gastrintestinais, reduziu o número de larvas infectantes recuperadas em coproculturas (Larsen et al. 1992). A redução de $\mathrm{L}_{3}$ de 0 . ostertagi no bolo fecal de bezerros, foi obtida alimentando-se os animais com grãos de cevada com adição de $D$. flagrans, porém obteve-se insucesso com a administração de grãos contendo $A$. oligospora (Grønvold et al. 1993, Wolstrup et al. 1994). Em uma menor quantidade de animais por área, a administração de material fúngico por três meses, demonstrou ser mais eficiente no controle no campo (Larsen et al. 1995). Ao se avaliar o efeito da administração de clamidósporos de $D$. flagrans 
no controle de Dictyocaulus viviparus e de alguns parasitos gastrintestinais, constatou-se menor preferencia pelos fungos em predar larvas de nematóides parasitos pulmonares (Fernández et al. 1999).

No Brasil, estudos com bovinos, em condições experimentais de campo, demonstraram que a administração oral de conídios de $A$. robusta, duas vezes por semana, durante quatro meses foi capaz de reduzir o OPG e a quantidade de vermes de animais traçadores infectados naturalmente com nematóides gastrintestinais (Araúijo et al. 1998).

\section{ADMINISTRAÇÃO DOS ISOLADOS SELECIONADOS}

Apesar do consenso que se criou em torno da produção de clamidósporos como forma ideal de administração dos isolados utilizados em programas de controle biológico, há falhas na tentativa em se desenvolver metodologia eficiente para a produção massal destas estruturas, assim como determinar se realmente apenas os clamidósporos são responsáveis pela capacidade dos fungos em se disseminar nas fezes e ambiente após passagem pelo trato gastrintestinal.

A passagem e recuperação de conídios de $A$. robusta, $A$. conoides e $M$. thaumasium na fezes de bovinos, demonstrou que conídios e micélio também foram capazes de atravessar o trato gastrintestinal dos animais e manter atividade predatória sobre larvas infectantes de $H$. placei, reduzindo a população deste nematóide, em comparação ao grupo controle (Araújo et al. 1996, 1999).

Um experimento, utilizando ovinos como animais experimentais, determinou, entre várias espécies de fungos testadas, que A. oligospora, Arthrobotrys oviformis e Geniculifera eudermata foram capazes de resistir ao estresse de passagem pelo trato gastrintestinal dos animais sem perda de capacidade predatória sobre larvas infectantes de $H$. contortus (Waller et al. 1994). A administração diária, por quatro meses de clamidósporos de $D$. flagrans administrados em grãos de cevada aos ovinos criados em sistema extensivo foi responsável pela conseqüiente redução da carga parasitária de Nematodirius spathiger, Nematodirius battus, no intestino delgado, além de Ostertagia spp e Trichostrongylus spp no abomaso (Githigia et al. 1997). Uma série de experimentos de passagem com vários isolados australianos, identificou quatro isolados de D. flagrans, como mais eficientes na passagem pelo trato gastrintestinal de ovinos (Faedo et al. 1997). Demonstrou-se também ser possível a passagem de clamidósporos de $D$. flagrans e de conídios de A. superba (Llerandi-Juárez \& Mendonza de Gives 1998).

A redução de larvas infectantes de ciatostomíneos em fezes de eqüinos também foi obtida com a administração de conídios de $A$. oligospora e $D$. flagrans em diferentes condições climáticas (Bird \& Herd, 1995, Santos et al. 1995, Baudena et al. 2000). O efeito de D. flagrans adicionado a suplemento alimentar na redução de Oesophagostomum dentatum e Hyostrongylus rubidus, parasitos de suínos foi observada, ao obter-se diminuição do parasitismo nos animais criados em sistema extensivo (Nansen et al. 1996).

\section{FORMULAÇÕES PARA UTILIZAÇÃO NO CONTROLE BIOLÓGICO DE NEMATÓIDES}

Até o presente momento, poucas tentativas para o desenvolvimento de formulações comerciais específicas para o controle de parasitos de animais utilizando estes fungos, obtiveram resultados promissores (Stirling et al. 1998). Em muitos estudos estes fungos têm sido produzidos em substratos sólidos como grãos de cereais e o substrato colonizado fornecido aos animais (Larsen et al. 1995, Waller et al. 2001). Algumas formulações comerciais destinadas ao controle de fitonematóides já foram desenvolvidas baseadas nesta forma de produção. Os produtos Royal 300 e Royal 350 foram desenvolvidos na França (Cayrol et al. 1978, Cayrol \& Frankowski 1979), baseados no cultivo de fungos do gênero Arthrobotrys em grãos de centeio. Entretanto, por desempenho inconsistente e problemas de controle de qualidade, foram pouco utilizadas e atualmente já foram retiradas do mercado.

Recentemente, formulações a base de alginato de sódio têm sido avaliadas experimentalmente no controle de nematóides parasitas de animais por alguns laboratórios de pesquisa. Esta formulação tem demonstrado bons resultados em condições laboratoriais e a campo (Araújo \& Sampaio 2000, Araúijo et al. 2000).

$\mathrm{O}$ fornecimento de material fúngico incorporado em blocos de suplementos minerais têm sido estudado na Austrália. Formulações minerais contendo clamidósporos do fungo D. flagrans têm obtido resultados positivos (Waller \& Faedo 1996). O obstáculo a este tipo de formulação se concentra na obtenção dos clamidósporos em grandes quantidades, pelo seu cultivo em grãos de cereais. Esta forma de produção é cara e dificulta o controle de qualidade do produto final (Stirling et al. 1998).

A adaptação de dispositivos intra-ruminal para liberação controlada de clamidósporos tem sido testada. Este mecanismo poderia ser aplicado em épocas estratégicas, liberando esporos por períodos de tempo prolongado, resultando em redução significativa no número de larvas infectantes nas pastagens. Foi avaliado um protótipo deste mecanismo, observando a presença de esporos viáveis em fezes de animais que receberam o dispositivo (Waller et al. 2001).

O desenvolvimento de formulações fúngicas para uso no controle biológico é um dos principais passos para a produção comercial destes microorganismos. Entretanto, não podemos nos esquecer do fator econômico. Deste modo, pesquisas que visam produzir material fúngico de maneira economicamente viável são extremamente necessárias e um passo importante para viabilizar a produção comercial de fungos nematófagos.

\section{PRESERVAÇÃO DA CAPACIDADE PREDATÓRIA DOS ISOLADOS SELECIONADOS COMO CONTROLADORES BIOLÓGICOS}

Apesar de todos os avanços realizados na pesquisa de utilização de fungos nematófagos como controladores biológicos de parasitos gastrintestinais de animais domésticos, alguns obstáculos impedem a sua completa implementação. As em- 
presas produtoras de fármacos anti-helmínticos são relutantes em investir em pesquisas emergentes, a menos que possuam a certeza de retorno de investimento e propriedade intelectual sobre os conhecimentos gerados. Deve-se desenvolver técnicas biológicas capazes de produzir grande quantidade de material fúngico em curto espaço de tempo e particularmente, na identificação taxonômica precisa dos agentes controladores (Waller \& Faedo 1996).

Como o critério de classificação dos fungos nematófagos está baseado em características morfológicas, várias revisões são publicadas tentando organizar de maneira mais lógica as espécies existentes. Para facilitar a produção em grande escala e dinamizar a rotina laboratorial, atendendo a necessidade de se trabalhar com pureza de espécies e evitar constantes contaminações das culturas, devem ser desenvolvidas metodologias capazes de preservar por período prolongado as características selecionadas dos fungos nematófagos para utilização em programas de controle biológico.

A preservação de culturas de microorganismos utilizados em programas de controle biológico é um pré-requisito para um grande número de procedimentos industriais e de pesquisa. Os critérios de escolha para um método específico de preservação recaem na manutenção de características morfológicas, bioquímicas e na estabilidade genética, pois caso os fungos sejam mantidos de modo a se adaptarem às condições de armazenamento, propriedades fundamentais dos isolados podem ser perdidas, ou ainda, técnicas que possibilitem apenas uma pequena taxa de sobrevivência de amostras podem resultar também na seleção de organismos variantes (Brown \& Gilbert 1995). Ademais, deve-se atentar para que o processo seja seguro e barato e ainda possibilite ao inóculo ser transportado, estocado e reconstituído com facilidade.

Há uma grande quantidade de métodos disponíveis para estoque e preservação de isolados fúngicos, organizados em três categorias: crescimento contínuo, secagem e suspensão do metabolismo. As técnicas de crescimento contínuo envolvem constantes transferências de um meio saturado para um meio nutritivo, propiciando ótimas condições de crescimento podendo ser estocadas em temperatura ambiente, refrigerador ou sob camada de água ou óleo mineral. As técnicas de secagem utilizam normalmente as formas de resistência dos fungos, como esporos, baseiam-se na redução da atividade de água e são caracterizadas por reduzir o metabolismo das células durante o período de armazenamento. Podem ser realizadas com sílica-gel, grãos de cereais e amostras de solo estéreis. Finalmente, há possibilidade de suspensão do metabolismo a níveis basais, por meio de técnicas de liofilização e manutenção em nitrogênio líquido, que propiciam redução de atividade de água por desidratação ou congelamento (Smith \& Onions 1994).

\section{PERSPECTIVAS FUTURAS}

Algumas perspectivas de pesquisa no uso de fungos para controle biológico de nematóides são: desenvolver formulações comerciais que contenham um ou mais isolados fúngicos, que sejam viáveis para fabricação em escala industrial e de fácil administração aos animais; realizar estudos de interação entre a cutícula da larva do nematóide e a parede celular dos fungos para caracterizar o mecanismo de adesão e penetração; testar os isolados com características predadoras promissoras em condições climáticas adversas; pesquisar outros modelos de interação entre novos parasitos e os isolados de fungos nematófagos com capacidade predatória comprovada; mensurar o impacto provocado pela introdução em grande escala de um ou mais fungos nematófagos no ambiente e avaliar métodos para retorno de capacidade predatória de isolados mantidos por períodos prolongados em condições laboratoriais.

\section{REFERÊNCIAS}

Araújo J.V. 1998. Capture of Cooperia punctata infective larvae by nematodetrapping fungi Arthrobotrys. Revta Bras. Parasitol. Vet. 7:101-105.

Araújo J.V. \& Sampaio W.M. 2000. Effects of temperature, mineral salt and passage through gastrointestinal tract of calves on alginate formulation of Arthrobotrys robusta. Revta Bras. Parasitol. Vet. 9:55-59.

Araújo J.V., Santos M.A., Ferraz S., Maia A.S. \& Magalhães A.C.M. 1992. Controle de larvas infectantes de Haemonchus placei por fungos predadores da espécie Monacrosporium ellypsosporum em condições de laboratório. Arq. Bras. Med. Vet. Zootec. 44:521-526.

Araújo J.V., Santos M.A., Ferraz S. \& Maia A.S. 1993. Antagonistic effect of predacious Arthrobotrys fungi on infective Haemonchus placei larvae. J. Helmintol. 67:136-138.

Araújo J.V., Neto A.P. \& Azevedo M.H.F. 1996. Screening parasitic nematodetrapping fungi Arthrobotrys for passage through the gastrointestinal tract of calves. Arq. Bras. Vet. Zootec. 48:543-552.

Araúijo J.V., Gomes A.P.S. \& Guimarães M.P. 1998. Biological control of bovine gastrointestinal nematode parasites in southern Brazil by the nematodetrapping fungus Arthrobotrys robusta. Revta Bras. Parasitol. Vet. 7:117-122.

Araújo J.V., Stephano M.A. \& Sampaio W.M. 1999. Passage of nematode-trapping fungi through the gastrointestinal tract of calves. Vet. Arhiv. 2:69-78.

Araúijo J.V., Sampaio W.M., Vasconcelos R.S. \& Campos A.K. 2000. Effects of different temperatures and mineral salt on pellets of Monacrosporium thaumasium - a nematode-trapping fungus. Vet. Arhiv. 80:181-190.

Balan J. \& Gerber N. 1972. Attraction and killing of the nematode Panagrellus redivivus by the predacious fungus Arthrobotrys dactiloides. Nematol. 18:163173.

Barger I.A. 1999. The role of epidemiological knowledge and grazing management for helminth control in small ruminants. Int. J. Parasitol. 29:4147.

Barron G.L. 1977. The Nematode-destroying Fungi. Topics in Mycobiology, No. 1. Canadian Biological Publications, Guelph, Canada. 140 p.

Baudena M.A., Chapman M.R., Larsen M. \& Klei T.R. 2000. Efficacy of the nematophagous fungus Duddingtonia flagrans in reducing equine cyathostome larvae on pasture in South Lousiana. Vet. Parasitol. 89:219230

Bird J. \& Herd R.P. 1995. In vitro assessment of two species of nematophagous fungi (Arthrobotrys oligospora and Arthrobotrys flagrans) to control the development of infective cyathostome larvae from naturally infected horses. Vet. Parasitol. 56:181-187.

Brown M.R.H. \& Gilbert P. 1995. Microbiological Quality Assurance. A guide towards relevance and reprodutcibility of inocula. CRC Press, Boca Raton, Florida. 337 p.

Cayrol J.C. \& Frankowski J.P. 1979. Une méthode de lutte biologique contre les nematodes à galles des racines appartenat au genre Meloidogyne. Revue Horticole 193:15- 23.

Cayrol J.C., Frankowsky J.P., Laniece A., D’Hardemare G. \& Talon J.P. 1978. Contre les nematódes en champignoniére. Mise au point d'une méthode 
de lute biologique a l'aide d'un hyphomicete prédateur: Arthrobotrys robustus souche antipolis (Royal 300). Revue Horticole 184:23-30.

Chandrawathani P., Omar J. \& Waller P. 1998. The control of free living stages of Strongyloides papillosus by the nematophagous fungus Arthrobotrys oligospora. Vet. Parasitol. 76:321-325.

Charles T.P., Rodrigues M.L.A. \& Santos C.P. 1995. Redução do número de larvas de Cyathostominae em fezes de eqüinos tratadas com conídios de Arthrobotrys oligospora. Arq. Bras. Med. Vet. Zootec. 47:87-89.

Cooke R.C. \& Godfrey B.E.S. 1964. A key of nematode-destroying fungi. Trans. Brit. Mycol. Soc. 47:61-74.

Coscarelli W. \& Pramer D. 1962. Nutrition and growth of Arthrobotrys oligospora. J. Bacteriol. 84:60-64.

Dackman C. \& Nordbring-Hertz B. 1992. Conidial traps - a new survival structure of nematode trapping fungus Arthrobotrys oligospora. Mycol. Res. 96:194-198.

Dackman C., Olsson S., Jansson H.B., Lundgren B. \& Nordbring-Hertz B. 1987. Quantification of predatory and endoparasitic nematophagous fungi and their activies in soil. Micro. Ecol. 13:89-93.

Descazeaux J. 1939. Action de champignons Hypomicétes prédateurs sur les larves de certains nématodes parasites des ruminants. Bull. Soc. Pathol. Exotique 32:457-459.

Descazeaux J. \& Capelle R. 1939. Contribuition à l'etude des champignons prédateurs des larves de nématodes parasites des animaux domestiques. Bull. Acad. Vét. France 12:284-288.

Dijksterhuis J., Harder W. \& Veenhuis M. 1993. Proliferation and function of the microbodies in the nematophagous fungus Arthrobotrys oligospora during growth on oleic acid or D-alanine as the sole carbon source. FEMS Microbiol. Letters 94:1-9.

Drechsler C. 1937. Some Hyphomycetes that prey on free living terricolous nematode. Mycologia 23:447-552.

Faedo M., Larsen M. \& Waller J.P. 1997. The potential of nematophagous fungi to control free-living stages of nematodes parasites of sheep: comparison between Australian isolates of Arthrobotrys spp and Dudingtonia flagrans. Vet. Parasitol. 72:149-155.

Fernández A.S., Larsen M., Nansen P., Grønvold J., Henriksen S.A., Bjørn H. \& Wolstrup J. 1999. The efficacy of two isolates of the nematode-destroying fungus Dudingtonia flagrans against Dictyocaulus viviparus larvae in faeces. Vet. Parasitol. 85:289-304.

Githigia S.M., Thamsborg S.M., Larsen M., Kyvsgaard N.C. \& Nansen P. 1997. The preventive effect of the fungus Duddingtonia flagrans on Trichostrongyle infections of lambs on pastutre. Int. J. Parasitol. 27:931-939.

Gray N.F. 1985. Ecology of nematode trapping fungi: effect on soil moisture, organic matter, distribution. Soil Biol. Bioch. 17:499-507.

Gray N.F. 1987. Nematophagous fungi with particular reference to their ecology. Biol. Rev. 62:245-304.

Griffin D.H. 1994. Fungal Physiology. Wiley-Liss, New York. 458 p.

Grønvold J. 1989. Induction of nematode-trapping fungus Arthrobotrys oligospora (Hyphomycetales), by infective larvae of Ostertagia ostertagi (Trichostrongilidae). Acta Vet. Scand. 30:77-87.

Grønvold J., Korsholm L., Wolstrup J., Nansen P. \& Henriksen S.A. 1985. Laboratory experiments to evaluate the ability of Arthrobotrys oligospora to destroy larvae of Cooperia species, and to investigate the effect of physical factors on the growth of the fungus. J. Helmintol. 59:119-125.

Grønvold J., Wolstrup J., Nansen P., Henriksen S.A., Larsen M. \& Bresciani J. 1993. Biological control of nematode parasites in cattle with nematode trapping fungi: a survey of danish studies. Vet. Parasitol. 48:311-325.

Grønvold J., Henriksen S.A., Larsen M., Nansen P. \& Wolstrup J. 1996. Aspects of biological control with special reference to arthropods, protozoans and helminths of domesticated animals. Vet. Parasitol. 64:47-64.

Jansson H.B. \& Nordbring-Hertz B. 1988. Infections events in the fungusnematode system, p. 59-62. In: Poinar O.G. \& Borne J.H. (ed.) Diseases of Nematodes. CRC Press, Boca Raton, Florida.
Larsen M. 1999. Biological control of helminths. Int. J. Parasitol. 29:139-146.

Larsen M., Wolstrup J. \& Henriksen S.A. 1992. In vivo passage through calves of nematophagous fungi selected for biocontrol of parasitic nematodes. J. Helmintol. 66:137-141.

Larsen M., Nansen P., Wolstrup J., Grønvold J., Henriksen S.A. \& Zorn A. 1995. Biological control of trichostrongylosis in grazing calves by means of the fungus Duddingtonia flagrans. Vet. Parasitol. 60:321-330.

Liu X. \& Zhang K. 1994. Nematode-trapping species of Monacrosporium with special reference to two new species. Mycol. Res. 8:862-868.

Llerandi-Juaréz D. \& Mendonza de Gives P. 1998. Resistance of nematophagous fungi chlamydospores to the digestive processes of sheep in Mexico. J. Helmintol. 72:155-158.

Macrae J.C. 1993. Metabolic consequences of intestinal parasitism. Proc. Nutr. Soc. 52:121-130.

Mendonza de Gives P., Zavaleta-Mieja E., Herrera-Rodrigues D. \& PerdomoRoldan F. 1992. Interaction between the nematode-destroying fungus Arthrobotrys robusta (Hyphomycetales) and Haemonchus contortus infective larvae in vitro. Vet. Parasitol. 41:101-107.

Mendonza de Gives P., Zavaleta-Mieja E., Herrera-Rodrigues D. \& QuirozRomero H. 1994. In vitro trapping capability of Arthrobotrys spp on infective larvae of Haemonchus contortus and Nacobbus aberrans. J. Helmintol. 68:223229.

Morgan-Jones G. \& Rodríguez-Kábana R. 1988. Infections events in the fungusnematode system, p. 59-62. In: Poinar O.G. \& Borne J.H. (ed.) Diseases of Nematodes. CRC Press, Boca Raton, Florida.

Mota M.A., Bevilaqua C.M.L. \& Araújo J.V. 2000. Atividade predatória de fungos Arthrobotrys conoides e Monacrosporium thaumasium sobre larvas infectantes de Haemonchus contortus de caprinos. Ciênc. Animal, Fortaleza, 10:37-41.

Nansen P., Grønvold J., Henriksen S.A. \& Wolstrup J. 1986. Predaceous activity of the nematode-destroying fungus Arthrobotrys oligospora on preparasitic larvae of Cooperia oncophora and on soil nematodes. Proc. Nematol. 53:237243

Nansen P., Foldager J., Hansen J.W., Henriksen S.A. \& Jørgensen R.J. 1988. Grazing and acquisition of Ostertagia ostertagi in calves. Int. J. Parasitol. 27:325-335.

Nansen P., Larsen M., Roepstorff A., Grønvold, J., Wolstrup J. \& Henriksen S.A. 1996. Control of Oesophagostomum dentatum and Hyostrongylus rubidus in outdoor-reared pigs by daily feeding with microfungus Duddingtonia flagrans. Parasitol. Res. 82:580-584.

Nordbring-Hertz B. \& Stälhammar-Carlemalm M. 1978. Capture of nematode by Arthrobotrys oligospora an electron microscope study. Can. J. Botany 56:1297-1307.

Pandey V.S. 1973. Predatory activity of nematode trapping fungi against the larvae of Trichostrongylus axei and Ostertagia ostertagi: a possible method of biological control. J. Helmintol. 47:35-48.

Perry B.D. \& Randolph T.F. 1999. Improving the assessment of the economic impact of parasitic diseases and their control in production animals. Vet. Parasitol. 84:145-168.

Pramer D. 1964. Nematode trapping fungi. Science 144:382-388.

Rew R.S. 1999. Production-based control of parasitic nematode of cattle. Int. J. Parasitol. 29:177-182.

Rosenweig W.D. 1983. Role of aminoacids, peptides and medium composition in trap formation by nematode-trapping fungi. Can. J. Microbiol. 30:265267.

Roubaud E. \& Descazeaux J. 1941. Action des Hyphomycetes prédateurs sur les larves de Synthétocaules et de Bunostomes. Bull. Soc. Pathol. Exotique 34:127-130.

Santos C.P., Charles T.P. \& Rodrigues M.L.A. 1995. Atividade predatória de Arthrobotrys oligospora e Duddingtonia flagrans em estádios pré-parasitários de ciatostomíneos em diferentes temperaturas. Revta Bras. Parasitol. Vet. 4:113-117. 
Saxena G. \& Mittal N. 1995. Trap formation by conidia of nematode-trapping Monacrosporium spp. Mycol. Res. 7:839-840.

Smith D. \& Onions A.H.S. 1994. The preservation and maintenance of living fungi. CAB International, Wallingford, United Kingdon. 122 p.

Stirling G.R., Smith L.J., Licastro K.A. \& Eden L.M. 1998. Control of root-knot nematode with formulations of the nematode-trapping fungus Arthrobotrys dactyloides. Biol. Contr. 11:224-230.

Stromberg B.E. 1997. Enviromental factors influencing transmission. Vet. Parasitol. 72:247-264.

Stromberg B.E. \& Averbeck G.A. 1999. The role of parasite epidemiology in the management of grazing cattle. Int. J. Parasitol. 29:33-39.

Thamsborg S.M., Poepstorff A. \& Larsen M. 1999. Integrated and biological control of parasites in organic and conventional production systems. Vet. Parasitol. 84:169-186.

Van Oorschot C.A.N. 1985. Taxonomy of the Dactylaria complex. A review of Arthrobotrys and allied genera. St. Mycol. 26:61-95.

Veenhuis M., Nordbring-Hertz B. \& Harder W. 1985. An electron microscopical analysis of capture and initial stages of penetration of nematodes by Arthrobotrys oligospora. Antonie Van Leeuwenhoek 51:385-398.

Waller P.J. \& Faedo M. 1996. The prospect for biological control of the freeliving stages of nematode parasite of livestock. Int. J. Parasitol. 26:915925.

Waller P.J., Larsen M., Faedo M. \& Henessy D.R. 1994. The potential of nematophagous fungi to control free-living stages of nematodes parasites of sheep: in vitro and in vivo studies. Vet. Parasitol. 51:289-299.

Waller P.J., Faedo M. \& Ellis K. 2001. The potential of nematophagous fungi to control the free living stages of nematodes parasites of sheep: towards the development of a fungal controlled release device. Vet. Parasitol. 102:321-330.

Wharton D.A. \& Murray D.S. 1990. Carbohydrate/lectin interactions between the nematophagous fungus Arthrobotrys oligospora and the infective juvenilles of Trichostrongylus colubrioformis (Nematoda). Parasitol. 101:101106.

Williams J.C. 1999. Integrated control (international experiences). Int. J. Parasitol. 29:183-184.

Wolstrup J., Grønvold J., Henriksen S.A., Nansen P., Larsen M., Bøgh, O. \& Iløe B. 1994. An attempt to implement the nematode-destroying fungus Duddingtonia flagrans in biological control of trichostrongyle infections of first grazing calves. J. Helmintol. 68:175-180.

Zhang K., Liu X. \& Cao L. 1996a. Nematophagous species of Monacrosporium from China. Mycol. Res. 100: 274-276.

Zhang K., Liu X., Cao L. \& Ren-Hen G. 1996b. A new species of Arthrobotrys from China. Mycol. Res. 100:527-530.

Zopf W. 1888. Zur Kenntnis der Infektions-Krankheiten niederer Thiere und Pflanzen. Nova Acta der Kaiserlichen Leopoldinischen-Carolinischen Akademie der Naturforscher 52:314-376. 